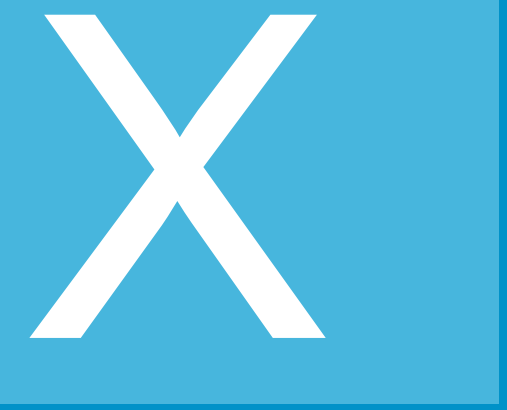

\title{
Estudio de la demanda de tasas de transferencia para determinar el ancho de banda requerido para el acceso a internet con calidad en instituciones universitarias
}

\section{Study of the demand for transfer rates to determine the bandwidth required for internet access with quality in universities \\ Universidad Nacional de San Martín, Perú}

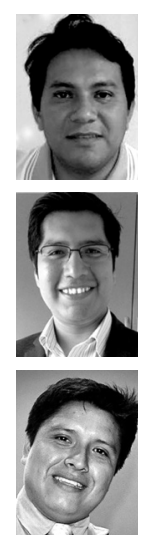

\section{Miguel Ángel Valles Coral}

Docente de la Universidad Nacional San Martín. Administrador de la Red de Fibra Óptica y del curso de Administración de Redes y Seguridad Informática en la FISI, y docente en la Universidad Peruana Unión, con maestría en Administración de Negocios y estudios concluidos de Maestría en Tecnologías de Información en la UNEG - Venezuela.

\section{Edison Effer Apaza Tarqui}

Ing. Edison Effer Apaza Tarqui. Docente de la Universidad Peruana Unión, Ingeniero en estadística e informática, especialista en estadística para la investigación, actualmente laborando como coordinador del Curso taller de Investigación en Ciencias Empresariales, en la Facultad de Ciencias Empresariales - Lima.

\section{Joel Pérez Suárez}

Ingeniero de sistemas por la Universidad Peruana Unión. Investigador y director de proyectos y patentes. Actualmente se desempeña como coordinador de la escuela de Ingeniería de Sistemas en la Universidad Peruana Unión. 
Miguel Ángel Valles Coral - Edison Effer

Apaza Tarqui - Joel Pérez Suárez

\section{Resumen}

Esta investigación es el resultado del estudio realizado a fines del año 2014 para determinar el ancho de banda mínimo requerido (a contratar) por la Universidad Nacional de San Martín - Tarapoto [UNSM-T], en función a las tasas de transferencia que a nivel de la red LAN demandan los estudiantes que cuenta con un dispositivo de conexión inalámbrica y lo utilizan en el campus universitario. Para determinar el ancho de banda requerido se aplicó la fórmula de Contreras, O y Contreras. N. (2010). Este cálculo se basó en la aplicación de métodos estadísticos que permiten determinar una muestra que permite inferir los resultados a la población universitaria.

Palabras clave: Ancho de banda, tasas de transferencia, demanda, cálculo.

\section{Abstract}

This research is the result of the study conducted at the end of the year 2014 to determine the minimum bandwidth required (to hire) by the Universidad Nacional de San Martín - Tarapoto [UNSM-T], in function to transfer to the LAN network-level rates require students who has a Wi-Fi device and use it on the University campus. To determine the required bandwidth applied the formula of Contreras, $\mathrm{O}$ and Contreras. N. (2010). This calculation was based on the application of statistical methods that allow to determine a sample that allows to infer the results to the University population.

Keywords: Bandwidth, transfer rates, demand, calculation. 
Estudio de la demanda de tasas de transferencia para determinar el ancho

\section{Introducción}

Las demandas de calidad en los servicios académicos de las instituciones superiores están relacionadas al servicio de internet. Entre las universidades que se encuentran en la región de San Martín, Perú, se encuentra la Universidad Nacional de San Martín (UNSM-T). Esta institución cuenta con una comunidad integrada por estudiantes, docentes, administrativos y público externo que esperan como mínimo contar con un servicio de internet de calidad a través de la red de datos disponible en la ciudad universitaria. Esto con el propósito de acceder a contenido educativo y bases de datos como consecuencia de la incorporación de la Tecnologías de Información (TI) en el proceso de enseñanza aprendizaje.

Paralelamente, los hábitos de los estudiantes acostumbrados en gran medida a contar con acceso de banda ancha de gran capacidad en su casa, representan un desafío para la calidad de servicio que la universidad puede ofrecer. Aproximadamente un $83.5 \%$ de alumnos cuentan con un equipo de acceso inalámbrico (Tablet, celular, etc.) que lo llevan al aula de clase o campus universitario, lo que genera una alta demanda del servicio de internet. Con esta información, es posible determinar el ancho de banda mínimo para proporcionar un servicio de calidad.

La oficina de Administración encargada de este servicio es la Red de Fibra Óptica de la UNSM-T, la cual mediante el estudio de la demanda de tasas de transferencia en la ciudad universitaria de la UNSM-T, determina el ancho de banda mínimo requerido para el acceso a internet con calidad.

\section{Materiales y métodos}

La ejecución del estudio se llevó a cabo en la ciudad universitaria de la UNSM-T, distrito de Morales, Provincia de San Martín, Perú. Asimismo, al estudio se aplicó una muestra de estudiantes de la UNSM-T, que cursan sus estudios en las diferentes carreras universitarias.

Para ello se aplicó el modelo matemático para la predicción del ancho de banda de Contreras, O y Contreras. N. (2010) que es "un modelo para la estimación de Ancho de Banda (BW) en Redes WAN considerando las aplicaciones, cantidad de usuarios y la simultaneidad de las conexiones. Para las aplicaciones se considera la carga máxima en el enlace y el aporte en relación al total de bytes transferidos. Para las simultaneidad de conexiones TCP/UDP se presentan funciones que permiten realizar estimaciones de ancho 
- Miguel Ángel Valles Coral - Edison Effer

Apaza Tarqui - Joel Pérez Suárez

de banda con una holgura cercana al $20 \%$ del canal disponible. Posteriormente se realizan pruebas del modelo estimando el BW y se efectúan comparaciones con datos observados de las estadísticas reales de Redes Corporativas", cuya fórmula propuesta es:

Donde:

$$
B W(b p s)=n * P_{A P}^{*} \varphi(n)
$$

$n=$ Número de Usuarios.

$\mathrm{P}_{\mathrm{AP}}=$ Peso de Aplicación. usuarios)

$P_{A P}=128000$ bps (basándonos en las aplicaciones que más usan los

La constante $\mathrm{P}_{\mathrm{AP}}$ representa el peso de la aplicación genérica usada para calcular los anchos de banda consumidos. Este valor es un promedio ponderado de los máximos de los protocolos más usados, a los cuales se les asignó un peso específico en función de su uso recurrente.

$\boldsymbol{\varphi}(n)=\mathbf{2 5 \%}$ Valor que se toma en función a la tasa de transferencia mínima considerada para una buena conexión y que el usuario. Por las características de la presente investigación, esta investigación es de tipo descriptiva.

Tabla 1

Universo y muestra determinada para el estudio

\begin{tabular}{lcc}
\multicolumn{1}{c}{ Facultad } & Universo & Muestra \\
\hline Ciencias Agrarias & 246 & 26 \\
Ciencias de la Salud & 493 & 52 \\
Ciencias Económicas & 1393 & 147 \\
Ingeniería Agroindustrial & 199 & 21 \\
Ing. Civil y Arquitectura & 805 & 85 \\
Ingeniería de Sistemas & 370 & 39 \\
Medicina Humana & 161 & 17 \\
Total general & $\mathbf{3 6 6 7}$ & $\mathbf{3 8 7}$ \\
\hline
\end{tabular}

Fuente: Elaboración propia.

\section{Resultados}

A continuación tablas de los resultados obtenidos en la investigación. 
Estudio de la demanda de tasas de transferencia para determinar el ancho

Tabla 2

Alumnos que cuentan con un dispositivo de conexión inalámbrica

\begin{tabular}{lrl}
\multicolumn{1}{c}{ Facultad } & \multicolumn{1}{c}{ NO } & \multicolumn{1}{c}{ sí } \\
\hline Ciencias Agrarias & $26.92 \%$ & $73.08 \%$ \\
Ciencias de la Salud & $13.46 \%$ & $86.54 \%$ \\
Ciencias Económicas & $17.01 \%$ & $82.99 \%$ \\
Ingeniería Agroindustrial & $4.76 \%$ & $95.24 \%$ \\
Ing. Civil y Arquitectura & $22.35 \%$ & $\mathbf{7 7 . 6 5 \%}$ \\
Ingeniería de Sistemas & $5.13 \%$ & $94.87 \%$ \\
Medicina Humana & $17.65 \%$ & $82.35 \%$ \\
Total general & $\mathbf{1 6 . 5 4 \%}$ & $\mathbf{8 3 . 4 6 \%}$ \\
\hline
\end{tabular}

Fuente: elaboración propia

Tabla 3

Alumnos que traen su dispositivo de conexión inalámbrica a la Ciudad Universitaria

\begin{tabular}{lll}
\multicolumn{1}{c}{ Facultad } & NO & sí \\
\hline Ciencias Agrarias & $42.11 \%$ & $57.89 \%$ \\
Ciencias de la Salud & $26.67 \%$ & $73.33 \%$ \\
Ciencias Económicas & $33.61 \%$ & $66.39 \%$ \\
Ingeniería Agroindustrial & $15.00 \%$ & $85.00 \%$ \\
Ing. Civil y Arquitectura & $28.79 \%$ & $71.21 \%$ \\
Ingeniería de Sistemas & $21.62 \%$ & $78.38 \%$ \\
Medicina Humana & $50.00 \%$ & $50.00 \%$ \\
Total general & $30.00 \%$ & $70.00 \%$ \\
\hline
\end{tabular}

Fuente: elaboración propia 
Miguel Ángel Valles Coral - Edison Effer

Apaza Tarqui - Joel Pérez Suárez

Tabla 4

Cálculo del ancho de banda a partir de los resultados del estudio

\begin{tabular}{lc}
\multicolumn{1}{c}{ Indicador } & Valor \\
\hline $\begin{array}{l}\text { Muestra } \\
\begin{array}{l}\text { Porcentaje de alumnos de la muestra que tienen } \\
\text { equipo de cómputo y lo llevan a la Ciudad Uni- } \\
\text { versitaria }\end{array}\end{array}$ & 387 \\
$\begin{array}{l}\text { Universo poblacional } \\
\text { Alumnos del universo poblacional que tienen }\end{array}$ & 3667 \\
$\begin{array}{l}\text { equipo de cómputo y lo llevan a la Ciudad Uni- } \\
\text { versitaria }\end{array}$ & 2142 \\
$\begin{array}{l}\text { Ancho de banda requerido con una tasa de } \\
\text { transferencia de 125 Kbps y una Calidad de } \\
\text { Servicio de 25\% }\end{array}$ & $\mathbf{6 6 . 9 3 ~ M b p s}$ \\
\hline
\end{tabular}

Fuente: elaboración propia

\section{Discusión}

La determinación de la velocidad de enlace a Internet que requieren los usuarios es $66.93 \mathrm{Mbps}$. Este indicador representa una tarea ardua que insta a determinar los servicios y/o prestaciones de Internet que necesita una empresa para el logro de sus objetivos. Asimismo existen otros factores que influyen, como la velocidad de transmisión de datos óptima en Internet, la misma que está de acuerdo a Hernández y otros (2009).

Se toma la fórmula propuesta por Contreras, O y Contreras. N. (2010), puesto que su trabajo presenta los antecedentes teóricos necesarios para desarrollar la línea de investigación el área del dimensionamiento y modelado de enlaces de telecomunicaciones, según sus propios autores y además porque se ajusta al modelo identificado en este estudio.

Por otro lado, las consideraciones tomadas en cuenta para evaluar la calidad de servicio se contrastan con lo afirmado por Frez (2011), ya que se realizaron mediciones que fueron relacionadas con métricas de desempeño y una evaluación de éstas respecto de la percepción de distintos servicios comúnmente utilizados, adoptándose entonces el indicador $\phi(n)=25 \%$ en función a la percepción adecuada de calidad de los usuarios según ese estudio. 
Estudio de la demanda de tasas de transferencia para determinar el ancho

\section{Conclusiones}

Este estudió realizó una estimación de la demanda de tasas de transferencia a partir de métodos estadísticos que permitieron inferir el porcentaje de estudiantes que cuentan con un equipo de cómputo.

Del mismo modo representa una estimación adecuada que permite hacer inferencias con los respectivos márgenes de error y representatividad, por lo tanto puede emplearse para el cálculo del ancho requerido para el acceso a internet con calidad.

A fin de proporcionar un servicio de calidad, es necesario establecer listas de control de acceso no muy restrictivas. Asimismo se recomienda que la UNSM-T, adquiera una línea de acceso a internet con al menos un $50 \%$ del valor obtenido en el presente cálculo. Tómese en consideración además que el cálculo realizado en esta investigación, se basa en la demanda que representan solo estudiantes, adicionalmente incluir la demanda de las oficinas académicas y administrativas.

Miguel Ángel Valles Coral Universidad Nacional de San Martín, Perú email: mavalles@unsm.edu.pe

Recibido: 12 agosto de 2015 Aceptado: 09 de diciembre de 2015 


\section{Miguel Ángel Valles Coral - Edison Effer \\ Apaza Tarqui - Joel Pérez Suárez}

\section{Referencias}

Camposano, D y Zambrano, G. (2013). Estudio del Ancho de Banda para el tráfico de Redes WAN de los ISP, con estudiantes de la Universidad Politécnica Salesiana Sede Guayaquil carrera Ingeniería de Sistemas, mediante la implementación de una página web. (Ingeniería de Sistemas GYE - Tesis de Pregrado). Universidad Politécnica Salesiana. Guayaquil - Ecuador.

Contreras, O y Contreras. N. (2010). Modelo Matemático para la Predicción de Ancho de Banda. Primera Aproximación. Artículo científico. Subgerencia de Administración y Operación de Redes - Ingeniería. Chile.

Hernandez, J y otros. (2009). Cálculo de ancho de banda necesario para una empresa. Universidad Juárez Autónoma de Tabasco. Tabasco - México. 4(2), 1-10. Recuperado de http://www. publicaciones.ujat.mx/publicaciones/revista_dacb/Acervo/v4n2OL/v4n2a1-ol/index. html

Frez, J. (2011). Estudio para el establecimiento de indicadores de calidad para el servicio de acceso a internet. (Tesis para optar al grado de magíster en ciencias mención computación). Universidad de Chile. Santiago de Chile - Chile. 